\title{
Nociones de libertad de expresión en disputa: la opinión pública publicada en la prensa ecuatoriana
}

\author{
Notions of freedom of expression in dispute: public opinion \\ published in the Ecuadorian press
}

Gustavo Eduardo Briones-Hidrovo gustavobh17@hotmail.com

http://orcid.org/0000-0001-6979-031X

Facultad de Periodismo y Comunicación Social; Universidad Nacional de La Plata (Argentina)

\section{Resumen}

El propósito de esta investigación es reconstruir los discursos publicados en la prensa ecuatoriana alrededor de la libertad de expresión, durante el periodo previo a la aprobación de la Ley Orgánica de Comunicación, en 2013.

Para el efecto, se seleccionaron artículos de opinión publicados en cuatro medios impresos de comunicación del Ecuador (El Telégrafo, El Comercio, El Universo y El Diario). Uno público, por ser el único de su índole y características; y tres privados, tomando en consideración su ubicación geográfica; de esta manera se busca mostrar distintos espacios de representación en la opinión pública. Se estudiarán, por tanto, los discursos para determinar las características del conflicto simbólico alrededor de la noción de libertad de expresión.

Palabras clave: discurso; análisis del discurso; prensa escrita; poder; opinión pública. 


\section{Abstract}

The purpose of this research is to reconstruct the discourses published in the Ecuadorian press around freedom of expression, during the period prior to the approval of the Organic Law of Communication, in 2013.

For this purpose, articles of opinion published in four print media of Ecuador (El Telégrafo, El Comercio, El Universo and El Diario) were selected. One public, for being the only one of its nature and characteristics; and three private ones, taking into account their geographical location; in this way, it seeks to show different spaces of representation in public opinion. Speeches will therefore be studied to determine the characteristics of the symbolic conflict around the notion of freedom of expression.

Keywords: discourse; discourse analysis; newspaper; press; power; public opinion.

La discusión y aprobación de Políticas Nacionales de Comunicación y Radiodifusión en distintos países latinoamericanos logró recolocar el interés por la problemática del poder de los medios en la opinión pública (Orlando, 2012). En el caso del Ecuador, que aprobó su Ley Orgánica de Comunicación en 2013, se pudo evidenciar que uno de los tópicos de dichas discusiones fue el derecho a la libertad de expresión.

Si bien el debate alrededor del derecho a la libertad de expresión emerge por el siglo XVI, es a fines del siglo XVII y durante el siglo XVIII que en los principales países de Europa y en Estados Unidos se establece el derecho del conjunto de la ciudadanía a la libertad de expresión, que de forma casi inmediata es ampliado a la divulgación de ideas y opiniones a través de los medios mecánicos, es decir, la libertad de prensa (Mastrini, Rossi y Califano, 2012: 11).

Este derecho, por tanto, se ve plasmado en legislaciones internacionales, desde la Declaración Universal de los Derechos Humanos, a mediados del siglo XX, y se extendió enfáticamente al periodismo al reconocer, en su artículo 19, el Derecho a la Información. Guarda una estrecha relación con principios considerados inherentes a la personalidad del hombre, entre los que se cuentan la libertad de opinión, de expresión y de prensa. El ejercicio de este derecho se realizaría a través de un mandato tácito a partir del cual el público delega la facultad de 
investigar, recibir y difundir informaciones en los periodistas y las empresas de medios, los cuales, a su vez, están obligados a cumplir con la misión social de informar (Loreti, 1995).

El derecho a la libertad de expresión se origina en las nociones occidentales básicas del papel individual en calidad de miembro de la sociedad (McQuail, 1992: 113). Por ende, este derecho requiere un debate público constante y regulación activa a fin de promover el acceso a los medios (Fiss, 1986). Desde esta concepción también llamada pluralista, "la libertad de expresión es tanto un derecho individual como colectivo y por ello se relaciona la calidad democrática con la mayor potencialidad expresiva de distintos actores sociales" (Becerra, 2015: 54). Loreti señala que son los medios de comunicación social los que sirven para materializar el ejercicio de la libertad de expresión, de tal modo que sus condiciones de funcionamiento deben adecuarse a los requerimientos de esa libertad (2015: 191-192).

Desde una concepción liberal, "históricamente, el Estado constituyó una amenaza para la libre circulación de ideas y opiniones y, por lo tanto, su intervención suele imponer obstáculos para la difusión de toda idea que el gobierno de turno considere inconveniente" (Becerra, 2015: 52). En concordancia con Becerra y Mastrini, los cambios en tema de políticas y/o derechos, así como de nuevos procesos de regulación en materia de comunicación en países como Argentina, Brasil, Venezuela, Bolivia y Ecuador, establecieron nuevas reglas del juego en cuanto a la relación de los medios de comunicación, la sociedad y el Estado (Kitzberger, 2010; Sorj, 2012).

En Ecuador, el gobierno de Rafael Correa puso en el centro de sus debates la acción de los medios en la reproducción simbólica del mundo social desde una posición cuestionadora a partir de tres aspectos principales: a. la vinculación de intereses entre las empresas mediáticas y los grupos económicos; b. la orientación política de la información en contra de su gobierno; y c. la impunidad de las malas prácticas periodísticas (Abad, 2012). Un estudio realizado por el Centro Internacional de Estudios Superiores de Comunicación para América Latina (CIESPAL) respecto de la cobertura mediática de la Ley de Comunicación en siete medios impresos ecuatorianos, realizado en 2012, revela que la información publicada estuvo dedicada a los temas: Consejo de Regulación (36,1\%); Libertad de expresión (22,9\%); Desvinculación económica (9,7\%); y Responsabilidad ulterior (8,3\%).

Al respecto del tratamiento periodístico del debate de la Ley Orgánica se puede determinar cómo estos medios impresos se convirtieron en actores políticos para confrontar el proyecto de Ley de ese entonces (Salazar, 2015). Para Abad (2012), el hecho de que un 22,9\% de las piezas analizadas en la investigación de CIESPAL giren en torno al tema "libertad de expresión" indica una fuerte tendencia de los medios a posicionar el tema dentro de ese marco 
interpretativo, y no en el de la lucha política por la reconfiguración del campo comunicacional e informativo en el Ecuador.

\section{El discurso de la prensa y la opinión pública}

Los medios de comunicación juegan un papel prioritario en las sociedades contemporáneas, a través de sus múltiples representaciones sociales y su capacidad de "construir realidades" (Vasilachis, 1997: 50). En el caso particular del periódico, este concentra un "formidable poder social" basado en "la capacidad de dar existencia explícita, de publicar, de hacer público, es decir, objetivado, visible, decible o, incluso, oficial a aquello que, al no haber accedido a la existencia objetiva y colectiva, continuaba en estado de experiencia individual o serial"; en otras palabras, en hacer sentido común (De Diego, 2011). A partir de esta noción se puede pensar el rol de la prensa como actor político y dispositivo de construcción de sentido. Entendiendo por actor político a "todo actor colectivo o individual capaz de afectar al proceso de toma de decisiones en el sistema político" (Borrat, 1989: 10).

Habermas sostiene que existen opiniones no públicas que son contrapuestas a la esfera de circulación de una opinión que denomina "cuasi pública". Sin embargo, estas opiniones formales pueden ser encausadas en instituciones como los medios de comunicación. La opinión pública para Habermas es una ficción en cierta manera necesaria para entender la realidad constitucional del Estado social como proceso. La define en relación con la manipulación de los dominadores para homogeneizar las disposiciones de la población a la doctrina y estructura política (1981: 261).

El modelo liberal de opinión pública se articula sobre la ficción, ya que se difunde la ideología que se presenta como defensora de los derechos de una sociedad liberal, entre ellos, la libertad de expresión. La considera ficción debido a que en la opinión pública se expresa la opinión "que se quiere hacer pública" (Adorno, 1964). Todo esto, es decir, la reproducción ideológica, la opinión pública y los medios de comunicación contribuyen en la formación de la opinión mediática. Precisamente, la opinión pública responde, no al entorno sino a un pseudoentorno construido por los medios informativos (McCombs, 2004). 


\section{La opinión pública publicada: análisis del corpus}

Los medios de comunicación han sido conceptualizados y, a la vez, naturalizados como herramientas que permiten difundir diversos sucesos sociales. Esto, acompañado de una serie de anuncios sobre su objetividad y neutralidad ha dotado de un fuerte poder de influencia sobre la creación de opinión y debate público, imaginarios sociales, discursos sociales y sobre las actuaciones de las personas (Sánchez, 2010).

Para realizar el análisis de la opinión pública publicada sobre la libertad de expresión, se utilizará el modelo tridimensional propuesto por Fairclough (1998), que considera tres niveles de análisis: el textual, el de la práctica discursiva y el de la práctica social; siendo el primero de carácter descriptivo, el segundo interpretativo y el tercero explicativo (Santander, 2011). Además, para analizar las prácticas sociales y discursivas en torno a las unidades de observación, se tomarán las herramientas teórico-metodológicas que propone Van Dijk (2011) a partir de la noción de discurso hegemónico.

El corpus de análisis consta de doce artículos de opinión de cuatro periódicos ecuatorianos: El Comercio, El Telégrafo, El Universo y El Diario, publicados entre el 2010 y 2013 . Si bien cada artículo seleccionado es un acto perteneciente a un micro nivel, es decir es ejecutado por un individuo, es parte constitutiva de un acto en el macro nivel ya que forma parte del discurso de un medio de comunicación masivo (Van Dijk, 1999).

Se eligieron cuatro medios impresos de comunicación del Ecuador. Uno público, por ser el único de su índole y características; y tres privados, tomando en consideración su ubicación geográfica. De esta manera se busca mostrar distintos espacios de representación en la opinión pública.

El Telégrafo fue creado en la ciudad de Guayaquil en 1984. En 2008 se refundó como periódico público. El Comercio, fue fundado en Quito en 1906. El Universo fue creado en la ciudad de Guayaquil en 1921 (Gehrke, 2016). En 2011, el entonces presidente, Rafael Correa, interpuso una demanda a título personal a tres directivos y un periodista de El Universo por "injurias calumniosas". El Diario, a diferencia de los periódicos anteriores, es un periódico de circulación provincial.

El corpus seleccionado (Tabla 1) se definió a partir de una serie representativa de artículos de opinión. Se delimitó su cantidad a partir de la noción de saturación teórica (Valles, 2005) centrado en el objeto discursivo "libertad de expresión". 


\begin{tabular}{|c|c|c|c|}
\hline \multicolumn{4}{|c|}{ Corpus de análisis (1) } \\
\hline EI Telégrafo & El Comercio & El Diario & EI Universo \\
\hline $\begin{array}{l}\text { (a) Gobierno defiende } \\
\text { libertad de expresión }\end{array}$ & (d) Acallar la voz & $\begin{array}{l}\text { (g) ¿Cuál libertad } \\
\text { de expresión? }\end{array}$ & $\begin{array}{l}\text { (j) Honor y libertad de } \\
\text { expresión de } \\
\text { funcionarios }\end{array}$ \\
\hline $\begin{array}{l}\text { (b) Debe haber } \\
\text { equilibrio }\end{array}$ & $\begin{array}{l}\text { (e) La falacia del } \\
\text { servicio público }\end{array}$ & $\begin{array}{l}\text { (h) Alfaro y la } \\
\text { libertad de } \\
\text { expresión }\end{array}$ & $\begin{array}{l}\text { (k) Libertad de } \\
\text { Expresión }\end{array}$ \\
\hline $\begin{array}{l}\text { (c) La cara oculta de } \\
\text { los medios }\end{array}$ & (f) La intolerancia & $\begin{array}{l}\text { (i) Libertad de } \\
\text { expresión }\end{array}$ & $\begin{array}{l}\text { (I) Manifiesto por la } \\
\text { libertad de expresión en } \\
\text { Ecuador }\end{array}$ \\
\hline
\end{tabular}

Tabla 1: Corpus seleccionado para realizar el análisis del discurso de la prensa ecuatoriana.

Fuente: elaboración propia a partir de artículos de opinión publicados en www.eluniverso.com; www.eltelegrafo.com.ec; www.eldiario.ec y www.elcomercio.com.

En lo que respecta al análisis textual se presenta en (a) un sujeto de la enunciación que se inserta al debate medios-Estado desde la elaboración de enunciados como "...libertad de expresión es la de todos los ciudadanos es muy diferente a la tan meneada libertad de expresión", "...se grita que el periodismo es sagrado y que la libertad de expresión es un recurso para difundir su verdad".

En (b), el sujeto de la enunciación utiliza tanto la primera persona del singular "...considero", “... he visto que muchos", para afirmar su posición, como la primera persona del plural para incluirse dentro de un grupo de individuos que está a favor de lo dicho en el enunciado "...obligarnos a reflexionar" "...a los lectores nos brinda la oportunidad", "...nos merecemos". Además, apela a un nosotros consciente al usar "...nos damos cuenta” de manera reiterativa. Por otra parte, utiliza la tercera persona del plural para referirse a la prensa y a los periodistas, en primera instancia con recursos tales como "....según dicen”, “...no han faltado quienes", “... algunos arguyen", “...ellos se creen”, para luego identificar a esa persona de la que se habla de manera directa como "...la prensa y los periodistas caen en esa crítica", "...gran poder de la prensa y de los periodistas", “...utilizan para manipular a la opinión pública”, “...buscan sembrar otra realidad lejos de la verdad en los lectores".

En (d) se utiliza la impersonalidad a lo largo del discurso y se toma como recurso la palabra "don" para referirse a la libertad de expresión "...la pérdida del don de la libertad de expresión cuando se apruebe la Ley de Comunicación”, “...evidentemente, equivale a haberlos colocado 
un bozal" Asimismo, usa adjetivos y adverbios modales para describir la considerada perdida “...toda esta terrible tragedia", “...lamentablemente”. En (e) también se recurre a este discurso en "...con esta perversa metamorfosis", "....actúan como curanderos idolatras".

A diferencia de (d), (f) usa un nosotros inclusivo "Vemos como el país anda totalmente desmoralizado y de tumbo en tumbo", pero coinciden en el uso de adjetivos y adverbios modales, “...Terrible violencia, "...tantísimas situaciones negativas”, “...lamentablemente”, “...un verdadero y real irrespeto a las diferentes opiniones y formas de pensar", así como construcciones lingüísticas como "...el país no ha vivido una época de respeto ni de expansión de la democracia, sino todo lo contrario", “...un verdadero y real irrespeto a las diferentes opiniones y formas de pensar" y “...comenzó a reinar la injusticia, la sinrazón, y, lo que es más, la falta de libertad, sobre todo la libertad de expresión".

En (e) se usa contenido informativo de la Ley y se argumenta sobre la expresión servicio público con enunciados como "...equivale a afirmar que la libertad de expresión de los ecuatorianos pasó de ser un derecho fundamental a realizarse como una actividad cuya titularidad es del Estado". El sujeto de la enunciación se refiere a libertad de expresión como "necesidad colectiva que requiere satisfacción", y legitima su discurso con "...la Constitución no prescribe que la comunicación sea un servicio público".

El sujeto de la enunciación en (g) se evidencia en la primera frase: "...me piden que hable de los periodistas". El tópico libertad de expresión del cual le piden hablar se vincula directamente con el ejercicio periodístico, a lo que refuta con enunciados como: "...libertad de expresión no es un derecho exclusivo de los periodistas", "...no se trata de que nosotros seamos quienes concedamos la libertad de expresión", “...es urgente repensar acerca de nuestras desmemorias, omisiones e incapacidades". El discurso atribuye más bien el derecho a la libertad de expresión a "la gente" desde construcciones lingüísticas como sinónimos de "silencio", "anonimato". Hace énfasis en su posición de periodista cuando enuncia "...cuando nuestro trabajo sea coherente con esa libertad de expresión".

En (h) se usa como referencia datos históricos acerca del "...establecimiento de la libertad de expresión" y cómo "...es un anacronismo tratar de regular el contenido de los que por ellos se difunde", haciendo referencia a los medios de comunicación. Visibiliza su postura asumiendo que "...los medios de difusión social del país siempre tienen conducta positiva", y se incluye en el colectivo que defiende al enunciar "...todos los comunicadores tenemos". Del mismo modo, en (i) se define la libertad de expresión como "... derecho humano fundamental y recurso para el desarrollo", y utiliza como recurso para la construcción de su enunciado "...nos unimos a 
esta protesta justa y solidaria" cuando se refiere al "...desafuero que se comete en contra de uno de los diarios más emblemáticos del país".

En el caso de (k) se utiliza un estilo más informal, refiere abiertamente a "...la polémica del presidente Correa contra los medios de comunicación", lo que advierte su posición ideológica. Sitúa su discusión no a los medios o comunicación o periodistas, sino a "la gente" que emite comentarios en redes sociales de estos medios "...me siento incómodo de que existan entes que regulen contenidos de los medios de comunicación en las redes sociales" y utiliza la palabra "consecuencias" al referirse a la Ley de Comunicación.

En el caso de (I) el sujeto de la enunciación se coloca en una posición de nosotros colectivo “...los escritores que firmamos este manifiesto" para indicar que está en contra del "... absurdo de responsabilizar a un medio y sus directivos por una opinión libre". Utiliza los términos "abuso de poder", "persecución”, "repudiamos", “indignación”. Establece su posición además con construcciones como ".... disentir activamente de toda forma de acoso y limitación a la libertad de expresión".

El sujeto de la enunciación de (j) hace referencia a acontecimientos ocurridos en el país en 2010 por lo que se inscribe en que "...el Ecuador necesita hoy más que nunca libertad de expresión e información". Se inscribe en una posición contraria con el uso de adjetivos como "lamentable" y construcción como "no nos rasguemos las vestiduras", el Ecuador sigue de tumbo en tumbo" "funcionarios imponiéndonos su visión de los hechos". "este Gobierno no entiende ni tolera la libertad de expresión".

Acerca de la práctica discursiva que se evidencia en los artículos analizados se puede interpretar lo siguiente. En el caso de (b) el contexto de discurso radica en un hecho suscitado en 2011 que fue considerado una cooptación explícita a la libertad de expresión por parte de la figura del presidente de la República, quien decidió enjuiciar a un medio de comunicación y a un periodista, a título personal, por "injurias calumniosas". El discurso en (b) hace precisamente una diferenciación entre periodista y medio de comunicación, situación que no ocurre en los demás artículos.

Una de las recurrencias encontradas en (b) fue la necesidad de asegurar que los lectores se dan cuenta de la posición de los medios de comunicación y de los periodistas, y que estos últimos hacen lo que el primero les ordena. Del mismo modo, utiliza recurrentemente el recurso del rumor, haciendo una analogía sobre el hecho de que los medios de comunicación manipulan la opinión pública.

El discurso de (c) radica en la crítica de los medios como empresas y del periodista como empleado mal asalariado. Para legitimar su discurso acerca del papel del periodista, (c) se 
basa en datos informativos de la Ley. En el caso de (a) se utiliza como recurso un hecho suscitado, el caso Assange, al igual que (b) para posicionarse en el discurso de que sí hay libertad de expresión y que el Gobierno la respalda y defiende.

Como se evidencia, los artículos (a), (b) y (c) poseen similares construcciones que dan vista de una posición a favor del gobierno y en contra de los medios de comunicación. Decir a favor del gobierno, es decir a favor de la libertad de expresión, y, en contra de los medios, no en todo el caso es en contra de los periodistas, pero siempre en contra de los medios, que para los enunciadores es el otro, el enemigo.

En contraposición, en la construcción de los titulares de (d), (e), (f), y (g) se transparenta un alejamiento o rechazo a las políticas gubernamentales. Una de las recurrencias encontradas en estos artículos es la utilización del término "régimen", en (e) puntualmente se hacen analogías de hechos actuales con "estilo fascista" y "dictadores", además de utilizar el término "poder político", determinan su posición de defensa a una libertad de expresión que está siendo coartada por el gobierno.

Se evidencia, además, la postura en el debate Estado-Medios, en una más abiertamente que en otros, en (I) por ejemplo, se expresa abiertamente la defensa a los medios de comunicación. En el caso de $(k)$ es el único que enuncia posterior a la aprobación de la Ley y se puede concluir la posición de su discurso cuando utiliza "consecuencias" para referirse a esta.

En suma, el uso de adjetivos es recurrente a lo largo de los discursos: malicioso, insultante, ofensiva, ruin, así como los tópicos poder, libertad de expresión, opinión pública, poder de la prensa, verdad. Se utilizan también, pero en menor medida, términos como "oligarquías", "poder político y económico"; además de adverbios modales como "lamentablemente".

Para explicar la práctica social visibilizada en los artículos analizados, se tomará además la propuesta de Van Dijk (2011) sobre el discurso ideológico, ya que plantea que la dimensión social de todo discurso se debe a que éste se lleva a cabo en situaciones específicas con el fin de conseguir actos de habla determinados, ya sea aserción, cuestión, acusación, promesa, amenaza, etc. De esta manera, la estrategia básica del discurso ideológico se basa en poner énfasis en "nuestros" aspectos positivos y en "sus" aspectos negativos (57-58)

Fairclough (1995) expone que el poder es entendido, por un lado, en términos de asimetrías entre los participantes de los eventos discursivos, y, por el otro, en tanto desigual capacidad de controlar cómo los textos son producidos, distribuidos y consumidos ( $\mathrm{y}$, en consecuencia, desigualdad en la forma de estos textos) en contextos socioculturales particulares. A partir de estas dos posturas de análisis, se puede decir que la homogeneidad de las posiciones discursivas a favor del gobierno, de El Telégrafo, permite articular un solo discurso 
repotenciado y legitimado al constituirse sobre varios relatos. Se visibiliza, además, una disputa de ideas en un espectro dualista en el cual se construye la idea del enemigo. Aquí existe una disputa por el sentido de la realidad, más específicamente por el sentido de una realidad política, en el que se expone al otro (medios de oposición) como un lugar ya constituido de fuerza (Gramsci, 2013), que intenta ejercer hegemonía contra otro grupo social (no dista de pertenecer a clases). Hay, además, una defensa y apropiación del sentido de libertad de expresión inherente al humano al desprenderlo de los mass medias, pero al mismo tiempo se lo arraiga a un gobierno que no necesariamente es entendido como proceso político.

Los periódicos El Diario, El Comercio y El Universo se sitúan en un espacio discursivo contrario de El Telégrafo. Los relatos discursivos contienen, en cambio, una idea homogénea de la construcción del "otro enemigo" y del espacio defendido, lo cual suma en la construcción de un discurso mediático que busca repotenciar su legitimidad con la pluralidad de voces.

Hay un distanciamiento del Estado, y más aún del gobierno de turno, con el cual se evidenciaría la tensión política y la existencia de voluntad de verdad (2). Además, se marca una separación de espacios duales, público y privado, siendo el primero el criticado.

Una de las centralidades de la construcción discursiva está en la crítica a la Ley de Comunicación, que es mostrada con una idea antidemocrática al usar el calificativo "Ley mordaza". Esta denominación fue creada y promovida por medios impresos ecuatorianos y, en palabras de Martín-Barbero (1978), esto no puede ser reducido a un discurso de relaciones de significación, sino a un problema de poder al interpretar a los medios como dispositivos (mass media). La adjetivación de la Ley buscó la producción y reproducción de un sentido ideológico (3) que promueva la participación de esos individuos en la lucha, para constituir una adhesión en los posicionamientos mediáticos. Si bien las voces discursivas participantes de estos medios construyen la idea de libertad de expresión desde aspectos subjetivos, ambiguos e interpretativos, se busca consolidar la idea de pluralidad de voces con base en un concepto de libertad contraria a la esencia de la "Ley mordaza". Está de por medio la disputa del sentido unidireccional del otro como enemigo.

A partir de este análisis, se puede comprobar cómo el poder de controlar el discurso es visto como el poder de sustentar prácticas discursivas particulares, asociadas a posturas ideológicas particulares, en un lugar de dominancia sobre otras prácticas alternativas (Fairclough, 2003). La tensión fluctúa en torno a la disputa por la construcción de "la verdad", al publicar opiniones con características recurrentes sobre la libertad de expresión según el medio. Estas luchas espectaculares muestran "el desarrollo desigual y conflictivo del sistema, los intereses relativamente contradictorios de las clases o subdivisiones de clases que aceptan el sistema, y 
definen la participación que les cabe en su poder" (Debord, 2005: 53). Por ejemplo, en El Telégrafo son los medios de comunicación y en ocasiones los periodistas y en El Comercio, el gobierno/presidente los que representan espacios de poder amenazados por el otro.

En los artículos de opinión no solo existe una crítica hacia el otro en la temática libertad de expresión, sino que hay un ejercicio "trinchera" desde donde estos elaboran su discurso y se identifican con el medio, lo que permite inferir un ejercicio de auto-legitimización por parte de los medios y su espacio, con la participación de estos individuos de opinión.

Existe una identificación entre quien opina y el medio en el que se expresa, en donde los medios, otorgan prestigio y realzan la autoridad de los individuos para buscar legitimidad de su status quo (Lazarsfeld, 1977). El discurso escrito por los individuos en cada medio denota una repetición de elementos e ideas que infiere un proceso hegemónico (4) discursivo que ha permeado en los lectores. Es así que se influye en decisiones de otros actores para que favorezcan los intereses y valores del actor que tiene el poder (Castells, 2012).

Los individuos centrales en la discusión sobre la temática "derecho de libertad de expresión" son una muestra de cómo usando inferencias o analogías, unido a una carga emocional convierte la construcción del discurso de un individuo en el discurso del medio en el que fue publicado. Estos individuos, podríamos decir, elaboraron su ejercicio discursivo alrededor de un acontecimiento, "que es el resultado de la movilización -que puede ser espontánea o provocada- de los medios alrededor de algo que, durante un cierto tiempo, éstos convienen en considerar como tal" (Champagne, 1999).

Los actores sociales que intervinieron expresando sus opiniones en cada medio se convierten en una forma de actuar social del grupo al que pertenecen (Van Dijk, 1999). Además, se evidencia que el rol del discurso dentro de la sociedad y la cultura es entendido como históricamente variable y que adquiere un rol esencial en la reproducción y en el cambio sociocultural (Fairclough, 2003). Es imprescindible darle la relevancia necesaria a analizar, por un lado, cómo las relaciones de poder restringen y controlan la productividad y la creatividad en las prácticas discursivas; y, por el otro, cómo una configuración particular de prácticas discursivas relativamente estables constituye un dominio hegemónico, ya que el poder de controlar el discurso es visto como el poder de sustentar prácticas discursivas particulares, asociadas a posturas ideológicas particulares, en un lugar de dominancia sobre otras prácticas alternativas (Fairclough, 1995: 2-5), por tanto, el discurso se vuelve no solo vehículo para expresar y reflejar ideas individuales. 


\section{Notas}

(1) Después del presente apartado se detalla la referencia de cada publicación que conforma el corpus de análisis de este trabajo.

(2) "...si el discurso verdadero ya no es, en efecto, desde los griegos, el que responde al deseo o el que ejerce el poder; en la voluntad de la verdad, en la voluntad de decir ese discurso verdadero, ¿qué es por tanto lo que está en juego sino el deseo y el poder?" (Foucault, 2015: 24).

(3) “...la instancia ideológica existe bajo la forma de 'formaciones ideológicas', que a su vez poseen un carácter 'regional' y comportan posiciones de clase: los objetos ideológicos son siempre proporcionados al mismo tiempo que 'la manera de servirse de ellos' -su 'sentido', es decir su orientación, es decir, los intereses de clase a los que sirven-, lo que se puede comentar diciendo que las ideologías prácticas son prácticas de clase... que se aplicarían luego a los diferentes 'objetos' ideológicos regionales de las situaciones concretas, en la Escuela, la Familia, etc." (Pecheux, 2016: 131).

(4) "El concepto político de 'hegemonía' puede emplearse de forma provechosa para analizar los órdenes del discurso (Fairclough, 1992; Forgacs, 1988; Laclau y Mouffe, 1985). Una particular estructuración social de la diferencia semiótica puede llegar a ser hegemónica, convirtiéndose en parte del sentido común legitimador que sustenta las relaciones de dominación, pero la hegemonía siempre será contrarrestada, en mayor o menor medida, mediante la lucha por la hegemonía" (Fairclough, 2001: 183).

\section{Corpus de análisis}

El gobierno defiende la libertad de expresión. (12 de octubre de 2012). El Telégrafo. Recuperado de https://www.eltelegrafo.com.ec/noticias/columnistas/1/gobiernodefiende-libertad-de-expresion

Díaz, J. (16 de abril de 2011). Debe haber equilibrio. El Telégrafo. Recuperado de https://www.eltelegrafo.com.ec/noticias/cartas-al-director/1/debe-haber-equilibrio

La cara oculta de los medios. (4 de enero de 2013). El Telégrafo. Recuperado de https://www.eltelegrafo.com.ec/noticias/columnistas/1/la-cara-oculta-de-los-medios

Acallar la voz. (19 de junio de 2012). El Comercio. Recuperado de http://www.elcomercio.com/cartas/acallar-voz.html

Zabala Egas, J. (28 de agosto de 2013). La falacia del servicio público. El Comercio. Recuperado de http://www.elcomercio.com/opinion/falacia-del-servicio-publico.html

La intolerancia. (5 de julio de 2012). El Comercio. Recuperado de http://www.elcomercio.com/cartas/intolerancia.html

Buitrón, R. (1 de mayo de 2010) ¿Cuál libertad de expresión? El Diario. Recuperado de http://www.eldiario.ec/noticias-manabi-ecuador/151238-cual-libertad-de-expresion/ 
Delgado Coppiano, R. (28 de mayo de 2011). Alfaro y la libertad de expresión. El Diario. Recuperado de http://www.eldiario.ec/noticias-manabi-ecuador/193129-alfaro-y-lalibertad-de-expresion/

Galarza, M. (30 de agosto de 2011). Libertad de expresión. El Diario. Recuperado de http://www.eldiario.ec/noticias-manabi-ecuador/202917-libertad-de-expresion/

Bertoni, E. (13 de junio de 2010). Honor y libertad de expresión de funcionarios. El Universo. Recuperado de https://www.eluniverso.com/2010/06/13/1/1363/honor-libertadexpresion-funcionarios.html

Martillo, J. (23 de junio de 2013). Libertad de expresión. El Universo. Recuperado de https://www.eluniverso.com/opinion/2013/06/23/nota/1058936/libertad-expresion

Manifiesto por la libertad de expresión en Ecuador. (25 de febrero de 2012). El Universo. Recuperado de https://www.eluniverso.com/2012/02/25/1/1355/manifiesto-libertadexpresion-ecuador.html

\section{Bibliografía}

Abad, O. G. (2012). La responsabilidad social del periodismo: procesos informativos, debates políticos y ejes de políticas públicas en el contexto de la Ley de Comunicación en el Ecuador. (Informe de Investigación). Universidad Andina Simón Bolívar, sede Ecuador, Quito, Ecuador.

Adorno, T. (1962). Prismas. Crítica de la cultura, crítica de la sociedad. Barcelona: Ariel.

Althusser, L. (1988). Ideología y aparatos ideológicos de Estado. Freud y Lacan. Buenos Aires: Ediciones Nueva Visión.

Becerra, M. (2015). De la concentración a la convergencia:medios, políticas y redes. Buenos Aires: Paidós.

Borrat, H. (1989). El periódico, actor político. Barcelona: Editorial Gustavo Gilli.

Castells, M. (2009). Comunicación y poder. Madrid: Alianza Editorial.

Champagne, P. (1999). La visión mediática. En Bourdieu, P. En la miseria del mundo (pp. 5163). Buenos Aires: Fondo de Cultura Económica.

De Diego, J. (mayo-julio 2011). Las luchas simbólicas del discurso de la prensa. Aportes de la perspectiva de Bourdieu al análisis del acontecimiento político. Razón y Palabra, 76. Recuperado de http://www.razonypalabra.org.mx/N/N76/varia/3a\%20entrega/33_Diego_V76.pdf 
De Moraes, D. (2011). La cruzada de los medios en América Latina. Gobiernos progresistas y políticas de comunicación. Buenos Aires: Paidós.

Debord, G. (2005). La sociedad del espectáculo (2 ed.). Barcelona: Pre-textos.

Fairclough, N. (1989). Languaje and power. Londres: Longman.

Fairclough, N. (1995). General Introduction. En N. Fairclough, Critical discourse analysis. The critical study of languaje (pp. 1-20). London and New York: Longman.

Fairclough, N. (2001). El análisis crítico del discurso como método para la investigación en ciencias sociales. En Wodak, R. y Meyer, M. (2003). Métodos de análisis crítico del discurso (pp. 179-203). España: Gedisa Editorial.

Fiss, O. (1997). Libertad de expresión y estructura social. México: Biblioteca de ética, filosofía del derecho y política.

Foucault, M. (2015). El orden del discurso. Argentina: Editores Tusquets.

Gehrke, H. et al. (2016). Panorama de los medios en Ecuador. Sistema informativo y actores implicados. Recuperado de http://m.dw.com/downloads/30336831/panorama-de-losmedios-en-ecuador-pdf.pdf

Gramsci, A. (2013). Antología. Buenos Aires: Siglo Veintiuno Editores.

Habermas, J. (1981). Historia y crítica de la opinión pública. España: Editorial Gustavo Gili.

Kitzberger, P. (noviembre de 2010). The Media Activism of Latin Americas's Leftist Governments: Does Ideology Matter? Recuperado de https://www.gigahamburg.de/en/system/files/publications/wp151_kitzberger.pdf

Lazarsfeld, F. (1977). La comunicación de Masas. Buenos Aires: Centro Editor de América Latina.

Loreti, D. (1995). El Derecho a la Información. Relación entre medios, público y periodistas. Buenos Aires: Editorial Paidós.

Loreti, D. y Lozano, L. (2015). El derecho a comunicar. Los conflictos en torno a la libertad de expresión en las sociedades contemporánea. Buenos Aires: Siglo XXI Editores.

Luhmann, N. (2007). La realidad de los medios de masas. Barcelona: Anthropos Editorial.

Martín-Barbero, J. (1978). Comunicación masiva: Discurso y poder. Ecuador: Editorial Época.

Mastrini, G. et al. (Ed.). (2012). Las políticas de comunicación en el siglo XXI: nuevos y viejos desafíos. Buenos Aires: La Crujía

McQuail, D. (1992). La acción de los medios. Los medios de comunicación y el interés públicos. Buenos Aires: Amorrortu.

McCombs, M. (2004). Estableciendo la agenda. El impacto de los medios en la opinión pública y en el conocimiento. Barcelona: Paidós. 
Muñoz, B. (2000). Theodor W. Adorno. Teoría Crítica y cultura de masas. Madrid: Fundamentos.

Orlando, R. (2012). Medios privados y nuevos gobiernos en Ecuador y Argentina. Quito: Flacso.

Pecheux, M. (2016). Las verdades evidentes. Lingüística, semántica filosofía. Buenos Aires: Ediciones del Centro Cultural de la Cooperación.

Sánchez, A. (2010). La acción de los medios de comunicación masiva en el Ecuador ¿acción comunicativa o estratégica? (Tesis de Maestría). FLACSO, Ecuador. Recuperado de https://bit.ly/2HRS9hZ

Santander, P. (2011). Por qué y cómo hacer Análisis de Discurso. Cinta moebio, 41, pp. 207224. Recuperado de www.moebio.uchile.cl/41/santander.html

Sorj, B. (2012). Democracia y Medios de Comunicación: Más allá del estado y el mercado. Buenos Aires, Argentina: Catálogos/Konrad Adenauer.

Valles, M. (2005). Metodología y tecnologia cualitativas: actualización de un debate, desde la mirada más atenta en la obra de Barney G. Glaser. Revista de Metodología de Ciencias Sociales, 9, pp. 145-168.

Van Dijk, T. (1999). Ideología. Una aproximación multidisciplinaria. México: Gedisa Editorial.

Van Dijk, T. (2011). Sociedad y discurso. Cómo influyen los contextos sociales sobre le texto y la conversación. México: Gedisa Editorial.

Vasilachis, de G. (1997). Construcción de representaciones sociales: discurso político y prensa escrita. Un análisis sociológico, jurídico y lingüístico. Barcelona: Gedisa Editorial. 